\title{
Adapting the contrast material protocol to the body surface area for an optimized low-dose CT coronary angiography with prospective ECG-triggering: a new evolving concept?
}

\author{
Thomas Hellmut Schindler
}

Received: 8 February 2010/ Accepted: 12 February 2010/Published online: 25 February 2010

(C) Springer Science+Business Media, B.V. 2010

CT coronary angiography (CTA) with the application of 64-slice CT scanners has been increasingly used for the non-invasive identification of subclinical $\mathrm{CAD}$, exclusion of morphologically focal epicardial lesions ( $<50 \%$ diameter stenosis) in individuals with a low-to-intermediate probability of the presence of $\mathrm{CAD}$, and for 3D image fusion with SPECT or PETdetermined myocardial perfusion imaging [1-5]. The diagnostic value of CTA in patients with high probability of the presence of $\mathrm{CAD}$ or clinically manifest $\mathrm{CAD}$, however, is limited due to coronary artery calcification (CAC)-induced blooming artifacts [5]. Another aspect is that the assessment of epicardial lesions still remains semiquantitative owing to limitations in spatial resolution and motion artifacts. Apart from these technical limitations, the relatively high radiation exposure for cardiovascular risk individuals during CTA has raised concerns for a more widespread clinical application [6, 7]. In this direction, however, the increasing use of prospective ECG-triggering or so called "snap-and-shoot mode",

Editorial comment on the article of Pazhenkottil et al. (doi:10.1007/s10554-010-9594-3).

T. H. Schindler $(\bowtie)$

Department of Internal Medicine, Cardiovascular Center, 6th Floor, Nuclear Cardiology, University Hospitals of Geneva, Rue Gabrielle-Perret-Gentil 4, 1211 Geneva, Switzerland

e-mail: thomas.schindler@hcuge.ch instead of conventional spiral image acquisition during CTA, has substantially reduced the radiation exposure of CTA from about $15-20 \mathrm{mSv}$ to $1-$ $3 \mathrm{mSv}$, while maintaining image quality and diagnostic accuracy [7, 8]. Similarly, ECG-controlled tube current modulation during helical CTA acquisition has also been shown to effectively reduce radiation exposure down to $5-7 \mathrm{mSv}[9,10]$.

In the present issue of the International Journal of Cardiovascular Imaging, Pazhenkottil et al. [11], report of a body surface area (BSA)-adapted contrast material $(\mathrm{CM})$ protocol for CTA, which resulted in a comparable coronary contrast enhancement independent of the individual BSA. This appears to represent an elegant approach to overcome, at least in part, a known variability in contrast attenuation of the coronary vessels [12] due to $\mathrm{CM}$ bolus dilution depending on the blood volume of the individual during CTA. As an increase in BSA is paralleled by an increase in blood volume, the authors tested whether a BSA-adapted CM protocol could compensate for the dilution of the CM depending on the blood volume of the individual person examined with CTA. An indeed, as it was observed in the present study [11], the range and standard deviation of the coronary attenuation were smaller in the BSAadapted protocol than in the group with standard $\mathrm{CM}$ protocol. These observations may suggest a well balanced and consistent attenuation of the coronary vessels throughout a large range of BSA. Whether such a BSA-adapted CM protocol results indeed in 
improved coronary vessel delineation as required for coronary plaque imaging remains uncertain.

Although CTA affords a reliable assessment of coronary artery plaque burden and arterial remodeling, an accurate qualitative and quantitative characterization of coronary plaque burden and its components has remained a challenge. Plaque attenuation thresholds may vary significantly with intracoronary lumen attenuation [13] and choice of reconstruction kernels $[12,14]$. The in the current study proposed BSAadapted CM protocol could be another important step in achieving a more consistent attenuation of the intracoronary lumen and, thereby, an improvement in the delineation of the arterial wall in individuals with different BSA or circulatory volume with CM dilution. On the other hand, it is equally possible that although the proposed BSA-adapted protocol achieves a more consistent attenuation of the coronary lumen as compared to a conventional rigid CM application, the intracoronary luminal contrast may not be sufficient to improve the delineation of the arterial wall as needed for imaging of coronary plaque burden. Thus, it remains a matter of ongoing research activities to define the best BSA-adapted CM protocol with an appropriate amount of $\mathrm{CM}$ that enables not only consistent intracoronary luminal attenuation but also an optimal delineation of the arterial wall for coronary plaque imaging. Such optimized CM protocols for CTA, as suggested by Pazhenkottil et al. [11], may lead to a further improvement in the identification and quantitative assessment of coronary plaque burden and its components providing an important framework to evaluate but also to monitor potential beneficial effects of primary or secondary preventive medical intervention on CAD process.

\section{References}

1. Haramati LB, Levsky JM, Jain VR et al (2009) CT angiography for evaluation of coronary artery disease in innercity outpatients: an initial prospective comparison with stress myocardial perfusion imaging. Int $\mathbf{J}$ Cardiovasc Imaging 25(3):303-313

2. van der Wall EE, Schuijf JD, Schalij MJ, Jukema JW, Bax JJ (2009) CT angiography; useful in non-selected outpatients? Int J Cardiovasc Imaging 25(3):315-318
3. von Kiedrowski H, Wiemer M, Franzke K et al (2009) Non-invasive coronary angiography: the clinical value of multi-slice computed tomography in the assessment of patients with prior coronary bypass surgery. Evaluating grafts and native vessels. Int $\mathbf{J}$ Cardiovasc Imaging 25(2):161-170

4. Bordeleau E, Lamonde A, Prenovault J et al (2007) Accuracy and rate of coronary artery segment visualization with CT angiography for the non-invasive detection of coronary artery stenoses. Int J Cardiovasc Imaging 23(6): 771-780

5. Raff GL, Gallagher MJ, O’Neill WW, Goldstein JA (2005) Diagnostic accuracy of noninvasive coronary angiography using 64-slice spiral computed tomography. J Am Coll Cardiol 46(3):552-557

6. Hausleiter J, Meyer T, Hadamitzky M et al (2006) Radiation dose estimates from cardiac multislice computed tomography in daily practice: impact of different scanning protocols on effective dose estimates. Circulation 113(10):1305-1310

7. Husmann L, Valenta I, Gaemperli O et al (2008) Feasibility of low-dose coronary CT angiography: first experience with prospective ECG-gating. Eur Heart J 29(2):191197

8. Husmann L, Herzog BA, Gaemperli O et al (2009) Diagnostic accuracy of computed tomography coronary angiography and evaluation of stress-only single-photon emission computed tomography/computed tomography hybrid imaging: comparison of prospective electrocardiogram-triggering vs. retrospective gating. Eur Heart $\mathbf{J}$ 30(5):600-607

9. Hausleiter J, Meyer T (2008) Tips to minimize radiation exposure. J Cardiovasc Comput Tomogr 2(5):325-327

10. Hausleiter J, Meyer T, Hermann F et al (2009) Estimated radiation dose associated with cardiac CT angiography. JAMA 301(5):500-507

11. Pazhenkottil A, Husmann L, Buechel R, et al. (2010) Validation of a new contrast material protocol adapted to body surface area for optimized low-dose CT coronary angiography with prospective ECG-triggering. Int J Cardiovasc Imaging (Epub ahead of print)

12. Herzog BA, Husmann L, Valenta I et al (2009) Determinants of vessel contrast in BMI-adapted low dose CT coronary angiography with prospective ECG-triggering. Int J Cardiovasc Imaging 25(6):625-630

13. Cademartiri F, Mollet NR, Runza G et al (2005) Influence of intracoronary attenuation on coronary plaque measurements using multislice computed tomography: observations in an ex vivo model of coronary computed tomography angiography. Eur Radiol 15(7):1426-1431

14. Cademartiri F, La Grutta L, Runza G et al (2007) Influence of convolution filtering on coronary plaque attenuation values: observations in an ex vivo model of multislice computed tomography coronary angiography. Eur Radiol 17(7):1842-1849 